\title{
hsa-miR-631 resensitizes bortezomib-resistant multiple myeloma cell lines by inhibiting UbcH10
}

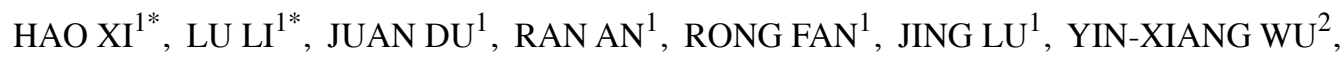 \\ SHU-XIE WU ${ }^{3}$, JIAN HOU ${ }^{1}$ and LI-MING ZHAO ${ }^{2}$
}

\begin{abstract}
${ }^{1}$ Department of Hematology, The Myeloma and Lymphoma Center, Changzheng Hospital, The Second Military Medical University, Shanghai 200003; ${ }^{2}$ Department of Respiratory Diseases, Eastern Hepatobiliary Surgery Hospital, The Second Military Medical University, Shanghai 200433; ${ }^{3}$ Grade Eight-Year Program, Xiangya School of Medicine, Central South University, Changsha, Hunan 410013, P.R. China
\end{abstract}

Received October 14, 2016; Accepted December 2, 2016

DOI: $10.3892 /$ or.2016.5318

\begin{abstract}
Although bortezomib (BTZ) remains a first-line agent for multiple myeloma (MM) therapy, the development of BTZ resistance has become an indicator of poor prognosis in MM patients. It is thus urgent to develop strategies to restore the vulnerability of MM to BTZ. This study demonstrated, for the first time, that $\mathrm{UbcH} 10$ is highly expressed in BTZ-resistant myeloma cell lines U-266/BTZ, NCI-H929/BTZ and RPMI-8226/BTZ, which is attributed to the inactivation of post-transcriptional control. The in-depth study revealed that during the development of BTZ resistance in these cells, the hsa-miR-631 levels were decreased, which resulted in the increased expression of the target gene UbcH10. We also found that the multiple drug-resistant protein MDR1 exhibited a positive correlation with $\mathrm{UbcH} 10$ due to the reduced ubiquitination of MDR1, which was caused by high UbcH10 expression. Following overexpression of miR-631, both BTZ sensitivity and BTZ-induced apoptosis were enhanced in the resistant cells. Meanwhile, resensitization by miR-631 overexpression was blocked by exogenous expression of UbcH10, which was not regulated by intracellular miR-631. In conclusion, the miR-631/UbcH10/MDR1 pathway is closely associated with the development of BTZ resistance in myeloma cells, and the
\end{abstract}

Correspondence to: Professor Jian Hou, Department of Hematology, The Myeloma and Lymphoma Center, Changzheng Hospital, The Second Military Medical University, 415 Fengyang Road, Shanghai 200003, P.R. China

E-mail: houjian@medmail.com

Professor Li-Ming Zhao, Department of Respiratory Diseases, Eastern Hepatobiliary Surgery Hospital, The Second Military Medical University, 700 North Moyu Road, Shanghai 200433, P.R. China E-mail: 1976zlm@163.com

${ }^{*}$ Contributed equally

Key words: hsa-miR-631, UbcH10, multiple myeloma, bortezomib, drug resistance overexpression of miR-631 can significantly improve BTZ sensitivity in resistant myeloma cells.

\section{Introduction}

Multiple myeloma (MM) is the second most common hematologic malignancy, with an incidence higher than that of acute leukemia. Although most MM patients are sensitive to initial chemotherapeutic treatments, relapse often occurs due to acquired drug resistance $(1,2)$. The introduction of bortezomib (BTZ) represented a breakthrough in the treatment of MM. Chemotherapy regimens based on BTZ have become first-line recommendations for MM patients with untreated, relapsed or refractory myeloma, including patients who are eligible for autologous hematopoietic cell transplantation $(3,4)$. BTZ resistance has a strong impact on the clinical effectiveness of MM treatments. Therefore, determining the process by which BTZ resistance develops in MM and searching for effective regimens to overcome resistance are critical research areas (5).

In the present study, we established three BTZ-resistant cell lines, U-266/BTZ, NCI-H929/BTZ and RPMI-8226/BTZ, by using gradual induction. We examined the mRNA and protein expression levels of $\mathrm{UbcH} 10$ in the resistant and their parental cells. The data showed that UbcH10 protein was significantly increased in the resistant cell lines while mRNA was slightly increased, suggesting an inactivation of the post-transcriptional regulation of UbcH10. Since microRNAs (miRNAs) are a typical mechanism for post-transcriptional regulation $(6,7)$, we searched for miRNAs with a binding site on the 3'-untranslated region (3'UTR) of UbcH10. We then quantitatively measured the miRNAs in the resistant and parental cells. The results indicated that hsa-miR-631 was negatively correlated with the UbcH10 protein. A luciferase reporter assay verified that hsa-miR-631 was able to bind to UbcH10-3'UTR and inhibit protein expression through the seed site. The results suggest that the inactivation of $\mathrm{UbcH} 10$ regulation by hsa-miR-631 may be a molecular mechanism for resistance in MM cell lines. This study examined whether hsa-miR-631 was abnormally expressed in BTZ-resistant MM cell lines, whether abnormal UbcH10 expression caused by abnormal hsa-miR-631 expression was a critical factor in BTZ resistance, whether MDR1 is 
involved in UbcH10-associated BTZ resistance and whether the overexpression of hsa-miR-631 may reduce or reverse BTZ resistance in MM cell lines.

\section{Materials and methods}

Cell culture. Three MM cell lines, U-266, NCI-H929 and RPMI-8226, were purchased from the Chinese Academy of Sciences Cell Bank (Shanghai, China). Cell lines were maintained in RPMI-1640 medium containing $10 \%$ fetal bovine serum (FBS) (both from Invitrogen, Carslbad, CA, USA). The viral packaging cell line 293T was purchased from American Type Culture Collection (ATCC; Manassas, VA, USA) and maintained in Dulbecco's modified Eagle's medium (DMEM) supplemented with 10\% FBS. All cells were incubated at $37^{\circ} \mathrm{C}$ in a humidified $95 \%$ air and $5 \% \mathrm{CO}_{2}$ incubator. U-266, NCI-H929 and RPMI-8226 cells were cultured in a semi-suspension and passaged by centrifugation; 293T cells were adherent and passaged by trypsin digestion.

Establishment and verification of BTZ-resistant MM cell lines. Three selected MM cell lines, U-266, NCI-H929 and RPMI-8226 were cultured with BTZ in a gradient of increased concentrations starting from $0.1 \mathrm{nM}$ and doubling every three passages, i.e., $0.1,0.2,0.4,0.8,1.6,3.2,6.4,12.8,25.6$ and $51.2 \mathrm{nM}$. Cells were cultured in a medium containing $51.2 \mathrm{nM}$ BTZ for two additional passages. These cell lines were named U-266/BTZ, NCI-H929/BTZ and RPMI-8226/BTZ. The resistant and parental cells were seeded in 96 -well plates at $1 \times 10^{5}$ cells per well, and medium containing BTZ was added at a final concentration of 1,2, 4, 8, 16, 32 and $64 \mathrm{nM}$ for a period of $48 \mathrm{~h}$. Cell Counting Kit-8 (CCK-8) assay was employed to determine cell inhibition by BTZ and to calculate the drug dose causing $50 \%$ growth inhibition $\left(\mathrm{IC}_{50}\right)$ values. Meanwhile, the resistant and parental cells $\left(1 \times 10^{6} \mathrm{each}\right)$ were harvested for total RNA and protein extraction, followed by real-time PCR and western blotting to measure the UbcH10 mRNA, hsa-miR-631 and UbcH10 proteins.

\section{Plasmid construction}

Construction of luciferase reporter vectors. Human UbcH10 3'-UTR (259 bp) was amplified from cDNA obtained through the reverse transcription of the total RNA of 293T cells, using the primers (Forward/Reverse) 5'-GCTCTAGAGAAACCTAC TCAAAGCAG-3' and 5'-GCTCTAGAACCACAGCTCAAGA TAAA-3'. The amplification parameters were 32 cycles of denaturation at $95^{\circ} \mathrm{C}$ for $10 \mathrm{sec}$, annealing at $58^{\circ} \mathrm{C}$ for $30 \mathrm{sec}$ and extension at $72^{\circ} \mathrm{C}$ for $30 \mathrm{sec}$. The product was then digested with $X b a \mathrm{I}$ and inserted into the pGL3-promotor vector (Promega Corp., Madison, WI, USA). The seed region was mutated by point mutation from 5'-CAGGTC-3' to 5'-TCGCAG-3'. The resulting vectors were named pGL-WT-UbcH10 and pGL-MT-UbcH10.

Construction of cDNA expression vector. The CDS sequence of human UbcH10 (NM_007019.2) was amplified using the primers (Forward/Reverse) 5'-GGAATTCGCCACC ATGGCTTCCCAAAACCGCG-3' and 5'-CGGGATCCTC AGGGCTCCTGGCTGGTG-3', which contained an EcoRI cutting site and Kozak sequence and a BamHI cutting site, respectively. The cDNA was prepared by reverse transcription of RNA isolated from 293 T cells. The PCR product was digested and cloned into a pcDH-CMV lentiviral expressing vector. Finally, the recombinant vector was named pcDHUbcH10.

Construction of the miR-631 expression vector. Human genomic DNA was extracted from 293T cells and used for amplification of the template of the precursor sequence of miR-631. The primers (Forward/Reverse) used were 5'-GGAATTCTGGC ATGCCATAGCAGCGCAG-3' and 5'-CGGGATCCCTCCCAT CTAAGCTTCCCAAAGTGT-3'. The PCR product was digested using EcoRI and BamHI, ligated into linear pCDH-EF1-GFP vector (System Biosciences, Mountain View, CA, USA) and transformed into DH5 $\alpha$ competent cells. The obtained vector was called the pmiR-631 vector. The products of the vectors were confirmed by DNA sequencing. Endotoxin-free DNA was prepared in all cases.

Lentivirus packaging. One day before transfection, 293T cells were seeded into $10-\mathrm{cm}$ dishes. Then, $2 \mu \mathrm{g}$ of each pmiR-631 or pcDH-UbcH10 vector and $10 \mu \mathrm{g}$ pPACK Packaging Plasmid Mix (System Biosciences) were co-transfected using Lipofectamine 2000 (Invitrogen) in accordance with the manufacturer's instructions. The medium was replaced with DMEM plus $1 \%$ FBS. Forty-eight hours later, the supernatant was harvested and cleared by centrifugation at $5,000 \mathrm{xg}$ at $4^{\circ} \mathrm{C}$ for $5 \mathrm{~min}$, and it was passed through a $0.45-\mu \mathrm{m}$ pore PVDF membrane. The virus titre was determined by gradient dilution. The packaged lentiviruses were named Lv-miR631 or Lv-UbcH10.

Verification of the binding site of hsa-miR-631 on UbcH10-3'UTR by luciferase reporter assay. TargetScan was used to predict the theoretic target (seed region) of miR-631 in mRNA sequence of UbcH10 (NM_007019.3). Chemically synthesized miR631-mimics, inhibitor and negative control (NC) were obtained from Shanghai Sangon Biotech Co., Ltd. (Shanghai, China).

293T cells were transfected with the miR631-mimics, inhibitor or NC as well as pGL-WT-UbcH10 and pGL-MT-UbcH10 using Lipofectamine 2000 according to the manufacturer's instructions. Forty-eight hours after transient transfection, the cells were harvested and luciferase assays were performed. The relative luciferase activities (ratios of firefly and renilla luciferase activity) of lysates were measured using a Dual-Luciferase Reporter system (Promega Corp.).

Effects of expression of miR-631 or UbcH10 on BTZresistance and MDR 1 expression in MM cells. The cells were divided into five groups: control, Lv-control, Lv-miR631, Lv-UbcH10, and Lv-miR631/Lv-UbcH10. The cell lines in logarithmic phase U-266/BTZ, NCI-H929/BTZ and RPMI-8226/ BTZ were seeded into 6 -well plates at $5 \times 10^{5}$ cells per well. Lentiviral solution at an MOI of 30 was added the next day. Seventy-two hours later, the infection efficiency was examined by observation of fluorescent markers. The cells were divided into three parts: one was seeded into 96-well plates and cultured in media containing various concentrations of BTZ (1-32 nM). After $48 \mathrm{~h}$, a CCK-8 assay was used to calculate the $\mathrm{IC}_{50}$ values of BTZ before and after genetic intervention. Next, other cells were cultured in a medium containing $25 \mathrm{nM}$ BTZ, and apoptosis was examined by double staining assay after $48 \mathrm{~h}$. The third group of cells was collected and subjected 
to extraction of total RNA and protein, followed by real-time PCR and western blotting to measure the miR-631 and UbcH10 RNAs and the UbcH10 and MDR1 proteins, respectively.

Assessment of cell viability and $I C_{50}$ values. The three cell lines and their genetically engineered lines were seeded in 96-well plates at $5 \times 10^{4}$ cells per well, and BTZ was added to a final concentration at $1,2,4,8,16$ or $32 \mathrm{nM}$ for a period of $48 \mathrm{~h}$, followed by a CCK- 8 assay for cell viability. Briefly, $10 \mu \mathrm{l} \mathrm{CCK}-8$ solution was added, and the cells were cultured under normal conditions for an additional $4 \mathrm{~h}$ before measuring absorbance at $450 \mathrm{~nm}$. The cell inhibition ratio was calculated, based on the $\mathrm{IC}_{50}$ values at $48 \mathrm{~h}$.

Detection of apoptosis. Seventy-two hours after viral infection, U-266/BTZ, NCI-H929/BTZ and RPMI-8226/BTZ cells were seeded into 6 -well plates at $1 \times 10^{5}$ cells per well in medium containing $10 \mu \mathrm{M} \mathrm{BTZ}$ and were cultured for $48 \mathrm{~h}$. The cells were collected and measured for apoptosis using flow cytometry (FACSCalibur) after treatment using the Annexin V-FITC Apoptosis Detection kit II (cat no. 556570) (both from BD Biosciences).

Effect of hsa-miR-631 overexpression on the half-life of $M D R 1$ protein in resistant MM cells. Seventy-two hours after viral infection, U-266/BTZ cells were re-suspended in RPMI1640 medium with $10 \%$ FBS, reseeded to 6-well plates at $2 \times 10^{5}$ cells per well and cultured overnight. Then, the medium was replaced with serum-free RPMI-1640 medium containing either $50 \mu \mathrm{M}$ MG132 or $100 \mu \mathrm{g} / \mathrm{ml}$ cycloheximide (CHX), and the cells were incubated under normal conditions. The cells were collected at $0,1,2,4$ and $8 \mathrm{~h}$ after the drug treatment, and total protein was extracted for MDR1 detection by western blotting.

Measurement of mRNA levels. Total RNA was isolated with TRIzol reagent (Invitrogen) according to the manufacturer's instructions. RNA was reverse transcribed into cDNA using M-MLV Reverse Transcriptase and oligo(dT) $)_{18}$ primer (both from Takara Bio, Inc., Otsu, Japan). The following specific primers (Forward/Reverse) were used in quantitative PCR of human UbcH10 and $\beta$-actin: UbcH10, 5'-AAGACCTGAGG TATAAGCTC-3' and 5'-CCACTTTTCCTTCAGGATGTC-3'; $\beta$-actin, 5'-CCTGTACGCCAACACAGTGC-3' and 5'-ATACT CCTGCTTGCTGATCC-3'. The lengths of the amplified products were 143 and $211 \mathrm{bp}$. Real-time PCR was performed using SYBR $^{\circledR}$ Premix Ex Taq ${ }^{\mathrm{TM}}$ kit and the TP800 system (both from Takara Bio, Inc.). cDNA from 200 ng total RNA was used as the template. The PCR reactions were conducted using 40 cycles of denaturation at $95^{\circ} \mathrm{C}$ for $10 \mathrm{sec}$, annealing at $60^{\circ} \mathrm{C}$ for $20 \mathrm{sec}$ and extension at $72^{\circ} \mathrm{C}$ for $20 \mathrm{sec}$.

To test the miR-631 levels, total RNA $(2 \mu \mathrm{g})$ was used for cDNA preparation with the M-MLV reverse transcription kit and specific primers: U6 snRNA (NM_001101.3), 5'-TAC CTTGCGAAGTGCTTAAAC-3'; and miR-631, 5'-GTCGTA TCCAGTGCGTGTCGTGGAGTCGGCAATTGCACTGGA TACGAGGAGA-3'. RNA contents were detected using fluorescent dye PCR (Takara Bio, Inc.) in accordance with the manufacturer's instructions. The following primers (Forward/ Reverse) were used for quantification of human U6 snRNA

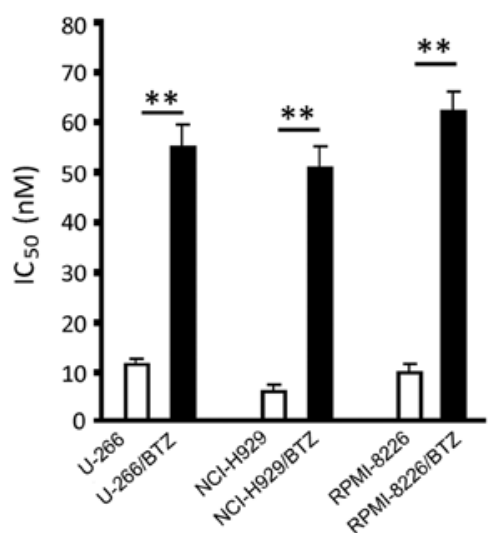

Figure 1. Verification of established BTZ-resistant cell lines. Cell viability of the MM parental and BTZ-resistant cells was assessed using CCK-8 assays in the presence of BTZ. The drug dose causing $50 \%$ growth inhibition $\left(\mathrm{IC}_{50}\right.$ drug dose) was calculated. Data are expressed as the mean \pm SD of at least three independent experiments. ${ }^{* *} \mathrm{P}<0.01$ relative to parental cells.

and miR-631: U6 snRNA, 5'-GTGCTCGCTTCGGCAGC ACAT-3' and 5'-TACCTTGCGAAGTGCTTAAAC-3', which produced a segment of $112 \mathrm{bp}$; and miR-631, 5'-GCCGGC GCCCGAGCTCTGGCTC-3' and 5'-AGACCTGGCCCAGAC CTCAGC-3', which produced a segment of $73 \mathrm{bp}$. The PCR systems included Takara SYBR Premix Ex Taq $10 \mu 1$, forward and reverse primers $(20 \mu \mathrm{M}) 0.2 \mu \mathrm{l}$ each, and cDNA $2 \mu \mathrm{l}$ added with $\mathrm{dH}_{2} \mathrm{O}$ to $20 \mu \mathrm{l}$. The cycling parameters were 40 cycles of denaturation at $95^{\circ} \mathrm{C}$ for $10 \mathrm{sec}$, annealing at $60^{\circ} \mathrm{C}$ for $20 \mathrm{sec}$ and extension at $72^{\circ} \mathrm{C}$ for $20 \mathrm{sec}$.

The mRNA levels of $\mathrm{UbcH} 10$ were normalized to the expression of an endogenous housekeeping gene, $\beta$-actin, using the $\Delta \Delta \mathrm{Ct}$ method. U6 snRNA was used as a reference to normalize the miR-631 levels using the $2^{-\Delta \Delta \mathrm{Ct}}$ method. Each RNA sample was run in triplicate.

Detection of proteins. Protein was extracted from the cells using M-PER mammalian protein extraction reagent (Pierce Biotechnology, Inc., Rockford, IL, USA). Equal amounts of protein (25 $\mu \mathrm{g}$ per lane), as estimated by a bicinchoninic acid (BCA) protein assay kit (Pierce Biotechnology, Inc.), were loaded onto (11\%) SDS-PAGE gels and transferred onto nitrocellulose membranes. The blots were probed with a monoclonal antibody against human UbcH10 (1:1,000), MDR1 (1:300) and $\beta$-actin $(1: 1,200)$, followed by the secondary HRP-conjugated anti-mouse/rabbit antibody (Santa Cruz Biotechnology, Inc., Santa Cruz, CA, USA). After washing, bands were detected by chemiluminescence and imaged with X-ray film, and relative optical densities were analyzed with the image processing software (TotalLab). Relative contents of proteins were calculated by dividing the optical density of the target band with the optical density of the $\beta$-actin band.

Data analysis. All data are expressed as the mean \pm SD and were analyzed by one-way ANOVA. Least significant difference (LSD) was used for multiple comparisons between any two means. P-values $<0.05$ were considered statistically significant. All statistical analysis was performed using SPSS 13.0 software. 
A

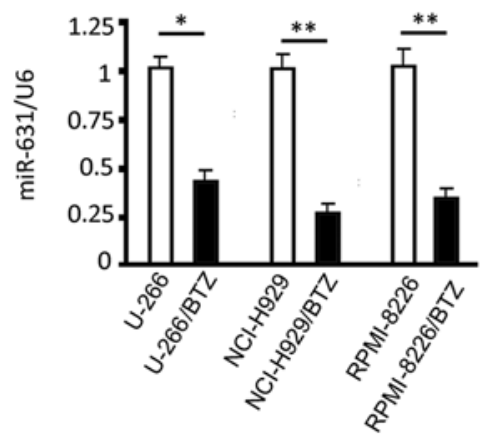

B

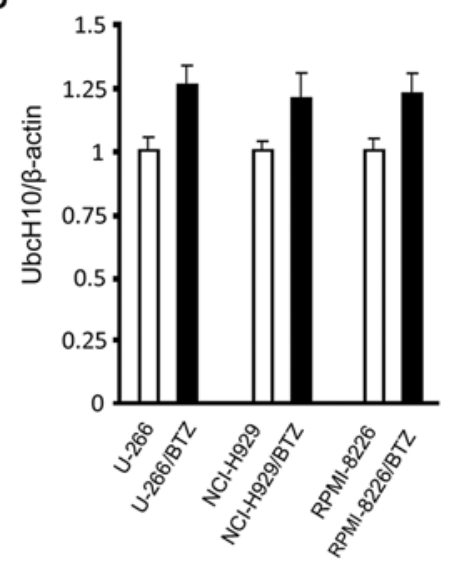

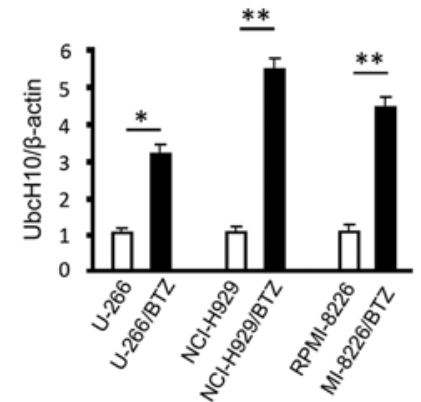

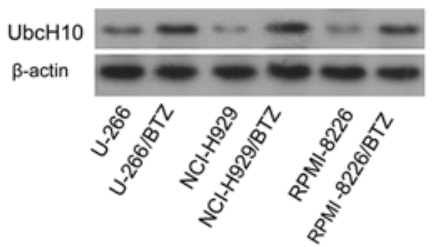

Figure 2. Expression of miR-631 and UbcH10 in BTZ-resistant cells. (A) miR-631 levels in the indicated cell lines measured by quantitative PCR. (B) mRNA and protein levels of UbcH10 expressed in the indicated cells determined using quantitative PCR and western blotting. Left, mRNA levels; Right, upper: optical density of the target band divided by the optical density of the $\beta$-actin band; and lower: representative blots. Data are expressed as the mean \pm SD of at least three independent experiments. ${ }^{*} \mathrm{P}<0.05$ and ${ }^{* * *} \mathrm{P}<0.01$ when compared to the control group.

\section{Results}

Establishment and confirmation of BTZ-resistant myeloma cell lines. We established the three BTZ-resistant cell lines U-266/BTZ, NCI-H929/BTZ and RPMI-8226/BTZ by gradually increasing the concentration of BTZ in the culture medium for $\sim 3$ months. The results of $\mathrm{IC}_{50}$ measurement showed that the $\mathrm{IC}_{50}$ values in the U-266/BTZ, NCI-H929/BTZ and RPMI-8226/BTZ cells increased from $11.10 \pm 1.24,6.08 \pm 0.71$ and $10.02 \pm 1.62 \mathrm{nM}$ in their parental cells to $55.62 \pm 4.88$, $49.12 \pm 4.32$, and $61.21 \pm 5.82 \mathrm{nM}$, respectively. These figures represented statistically significant differences between the resistant cells and their parental cells $(\mathrm{P}<0.01)$ (Fig. 1).

Examination of miR-631 and UbcH10 expression in resistant myeloma cells. Quantitative results showed that the miR-631 levels in resistant myeloma cells were significantly lower than those in the parental cells $(\mathrm{P}<0.05)$ (Fig. 2A). Western blotting results indicated that the Ubc10 protein levels were higher in the resistant myeloma cells than these levels in the parental cells $(\mathrm{P}<0.05)$ (Fig. 2B). While UbcH10 mRNA increased in the resistant cells, there was no significant difference between the resistant cells and their parental cells ( $\mathrm{P}>0.05)$ (Fig. 2B).

Prediction of hsa-miR-631 binding site in UbcH10-3'UTR and verification by luciferase reporter assay. The TargetScan analysis demonstrated a theoretic binding site (seed region) 5'-CAGGUC-3' in the 3'UTR region of the UbcH10 gene from bases 72-78. We cloned 3'UTR of UbcH10 into the pGL-3 promoter luciferase reporter vector for verification purposes. Luciferase activity detection indicated that the miR631-mimic significantly inhibited intercellular luciferase activity ( $\mathrm{P}<0.05$, compared to the group transfected with luciferase expression vector alone), and that miR631 inhibitor slightly increased the luciferase activity without reaching statistical
A

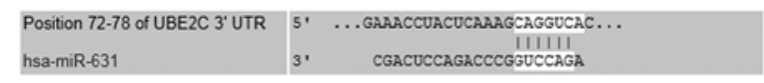

B

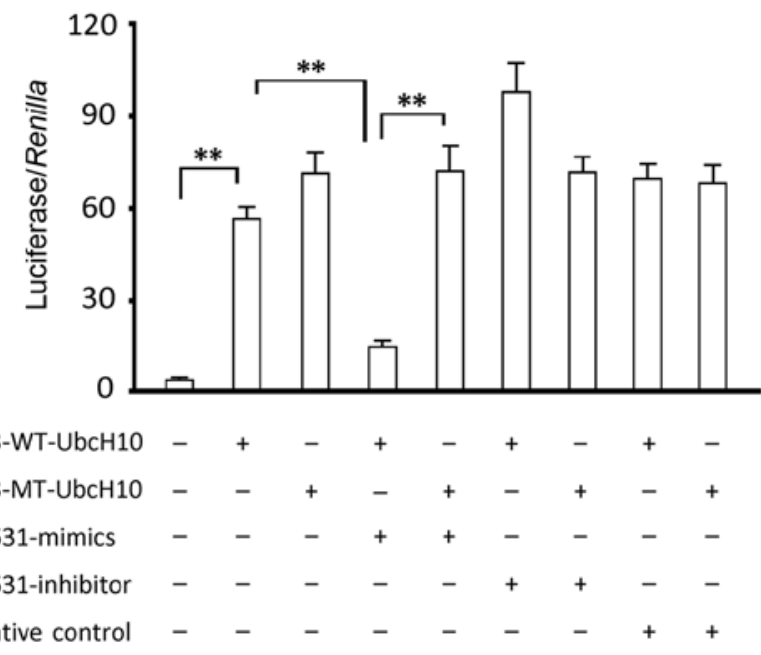

Figure 3. Verification of the interaction between miR-631 and UbcH10. (A) Predicted binding site of miR-631 in the 3'UTR region of the UbcH10 gene. (B) $293 \mathrm{~T}$ cells transfected with pGL3-WT-UbcH10 or pGL3-MTUbcH10 and with or without miR631-mimic or miR631-inhibitor. The histogram indicates relative firefly luciferase activities in the different transfected groups. Error bars represent standard deviation and were obtained from at least three independent experiments. ${ }^{* *} \mathrm{P}<0.01$.

significance (Fig. 3). However, neither produced a change in luciferase activity in cells transfected with the luciferase expression vector carrying a mutated binding site. Compared to the group transfected with only luciferase expression vector, cells transfected with miR631-NC showed a similar luciferase activity, indicating that RNA transfection had no effect on luciferase activity. These data suggest that the binding site of miR-631 in UbcH10 aligns with the predicted sequence. 
A
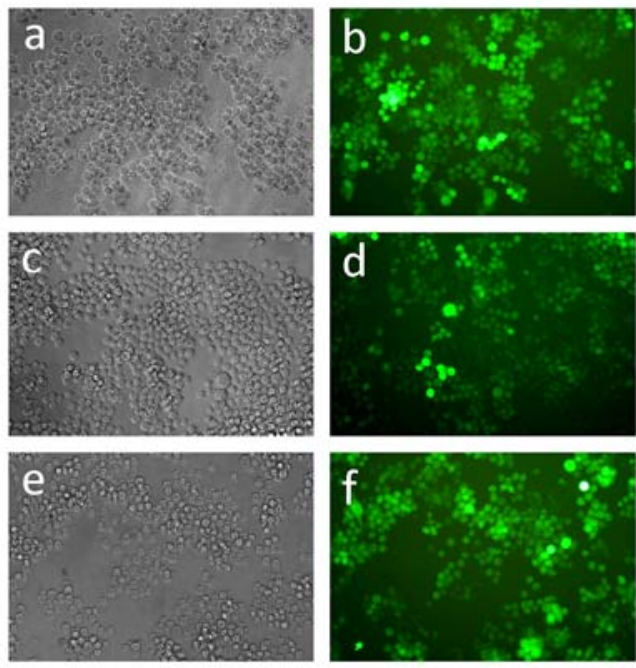

B

U-266/BTZ
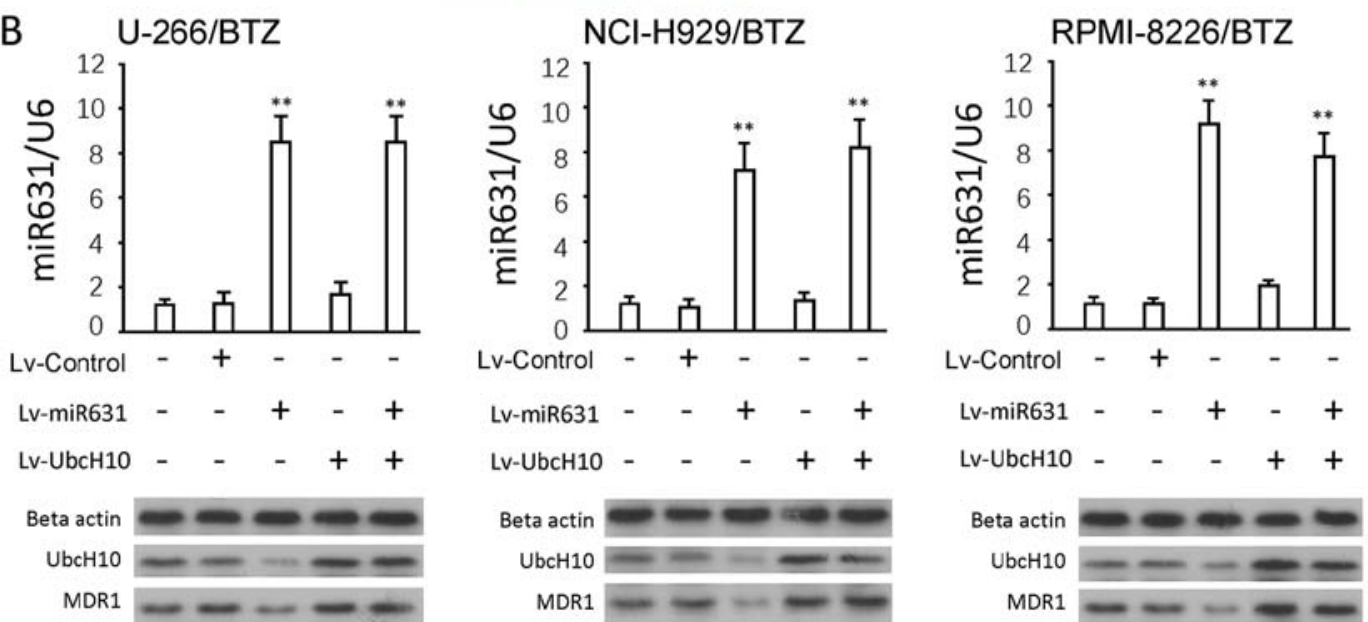

Figure 4. Overexpression of miR-631 and UbcH10 in BTZ-resistant cells. (A) U-266/BTZ, NCI-H929/BTZ and RPMI-8226/BTZ cells were infected with lentivirus and subjected to fluorescence microscopy to observe GFP. (B) RNA and proteins were extracted and measured for miR-631, UbcH10 and MDR1 proteins using real-time PCR and western blotting after $72 \mathrm{~h}$. Upper, miR-631 levels assessed by quantitative PCR. Lower, representative blots of UbcH10 and MDR1. Data are expressed as the mean $\pm \mathrm{SD}$ of at least three independent experiments. ${ }^{* *} \mathrm{P}<0.01$ compared to control groups.

Overexpression of $\mathrm{miR}-631$ and $\mathrm{UbcH} 10$ in resistant cells using a lentiviral approach. Genetic intervention was conducted in the three resistant cell lines using a lentiviral approach. The gene delivery efficiency was close to $100 \%$, according to the GFP levels (Fig. 4A). Lv-miRNA631 infection significantly increased mature miR-631 levels in both resistant cell lines and parental cells ( $\mathrm{P}<0.01)$ (Fig. 4B). Western blotting results demonstrated that Lv-miR631 significantly decreased UbcH10 protein ( $\mathrm{P}<0.01$ vs. control). Lv-UbcH10 infection significantly increased $\mathrm{UbcH} 10$ protein $(\mathrm{P}<0.01$ vs. control), as did the combination of Lv-miR631 and Lv-UbcH10. No obvious difference was found in these groups compared to the Lv-UbcH10 infection group $(\mathrm{P}>0.05)$. The changes in the MDR1 proteins were similar to those found in $\mathrm{UbcH} 10$.

Measurements of $I C_{50}$ values and apoptosis. The overexpression of miR-631 significantly decreased the $\mathrm{IC}_{50}$ values of the resistant cells to BTZ $(\mathrm{P}<0.01)$, whereas $\mathrm{UbcH} 10$ overexpression slightly increased the $\mathrm{IC}_{50}$ values, with no significant difference $(\mathrm{P}>0.05)$. The overexpression of both miR-631 and UbcH10 significantly increased the $\mathrm{IC}_{50}$ values $(\mathrm{P}<0.01$, vs. control), with no difference in the $\mathrm{UbcH} 10$ overexpression group ( $\mathrm{P}>0.05)$ (Fig. 5A).
The apoptosis rate (including early apoptosis and late apoptosis) of the U-266/BTZ cells receiving genetic interventions and undergoing the treatment of $25 \mathrm{nM} \mathrm{BTZ}$ for $48 \mathrm{~h}$ were as follows (Fig. 5B): control, 24.63 $\pm 3.02 \%$; Lv-Control, $23.96 \pm 2.87 \%$; Lv-miR631, 86.13 $46.45 \%$; Lv-UbcH10, $18.73 \pm 3.92 \%$; Lv-miR631 and Lv-UbcH10, 19.97 $\pm 2.14 \%$. The apoptosis rate of the Lv-miR631-infected resistant cells was significantly increased than the rates observed in the other four groups $(\mathrm{P}<0.01)$, and there were no differences between the other four groups $(\mathrm{P}>0.05)$. The effects of genetic intervention on IC $_{50}$ values in the NCI-H929/BTZ and RPMI-8226/ BTZ cells coincided exactly with those in the U-1996/BTZ cells (data not shown).

Effect of hsa-miR-631 overexpression on the half-life of MDRI protein in $U-266 / B T Z$ cells. When MG132 was used to inhibit protein degradation, MDR1 increased from 0 to $8 \mathrm{~h}$, while the rate of increase between the groups showed no difference. The use of CHX to inhibit protein synthesis resulted in a decrease in MDR1 from 0 to $8 \mathrm{~h}$. The reduction in MDR1 protein in the Lv-miR631 group was more rapid than that in the control group (Fig. 6). 

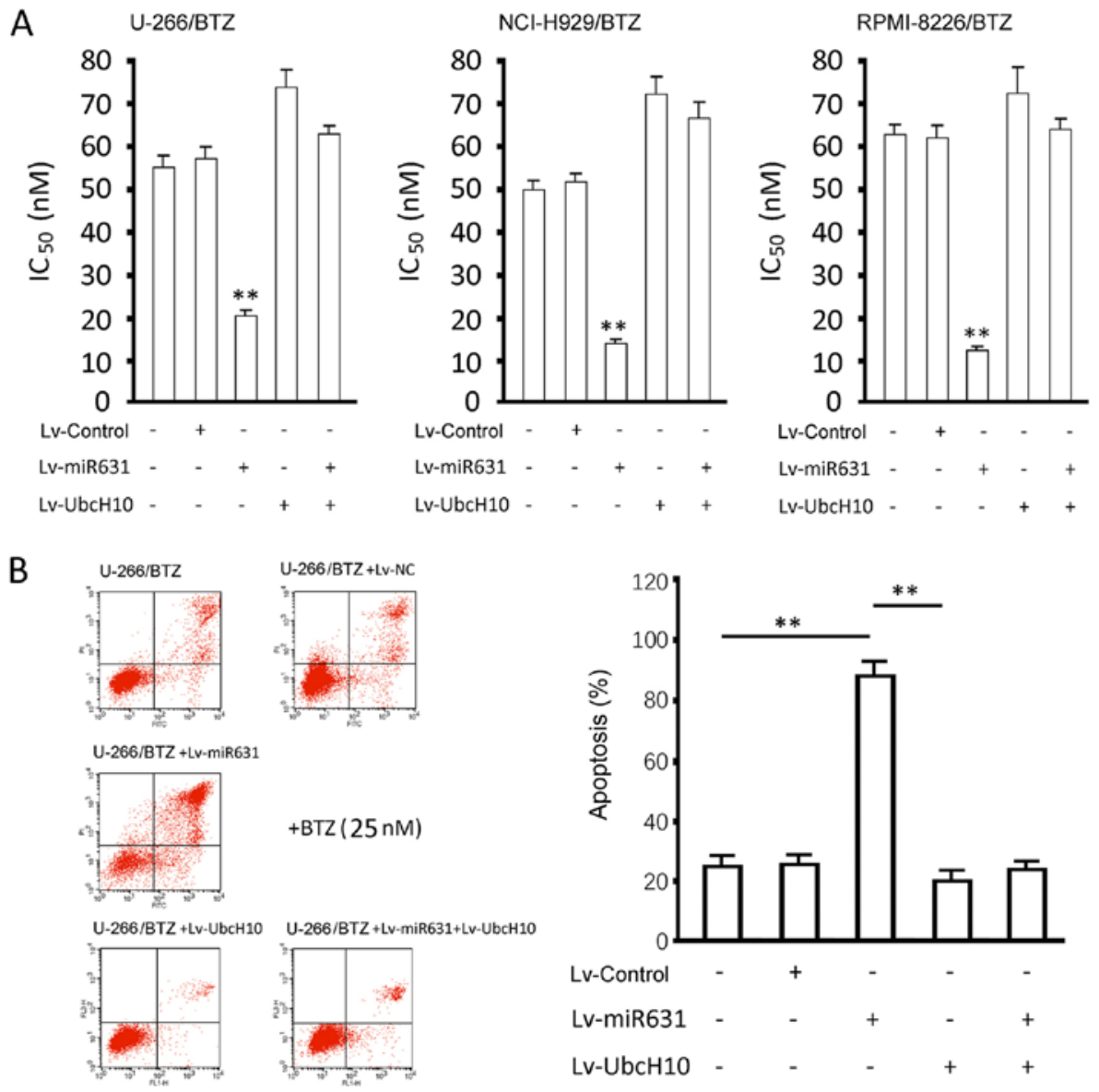

Figure 5. $\mathrm{IC}_{50}$ values and apoptosis in resistant cells overexpressing miR-631 and UbcH10. (A) Cell viability of the BTZ-resistant cells infected with the indicated lentivirus was assessed using CCK-8 assays in the presence of BTZ. The drug dose causing $50 \%$ growth inhibition ( $\mathrm{IC}_{50}$ drug dose) was calculated. ${ }^{* *} \mathrm{P}<0.01$ relative to the control cells. (B) Apoptosis in U-266/BTZ cells. Left panel, representative plots of U-266/BTZ cells with the indicated treatments. Right panel, quantification of apoptosis for the indicated treatments. Data represent means \pm SD of at least three separate experiments. ${ }^{* *} \mathrm{P}<0.01$.
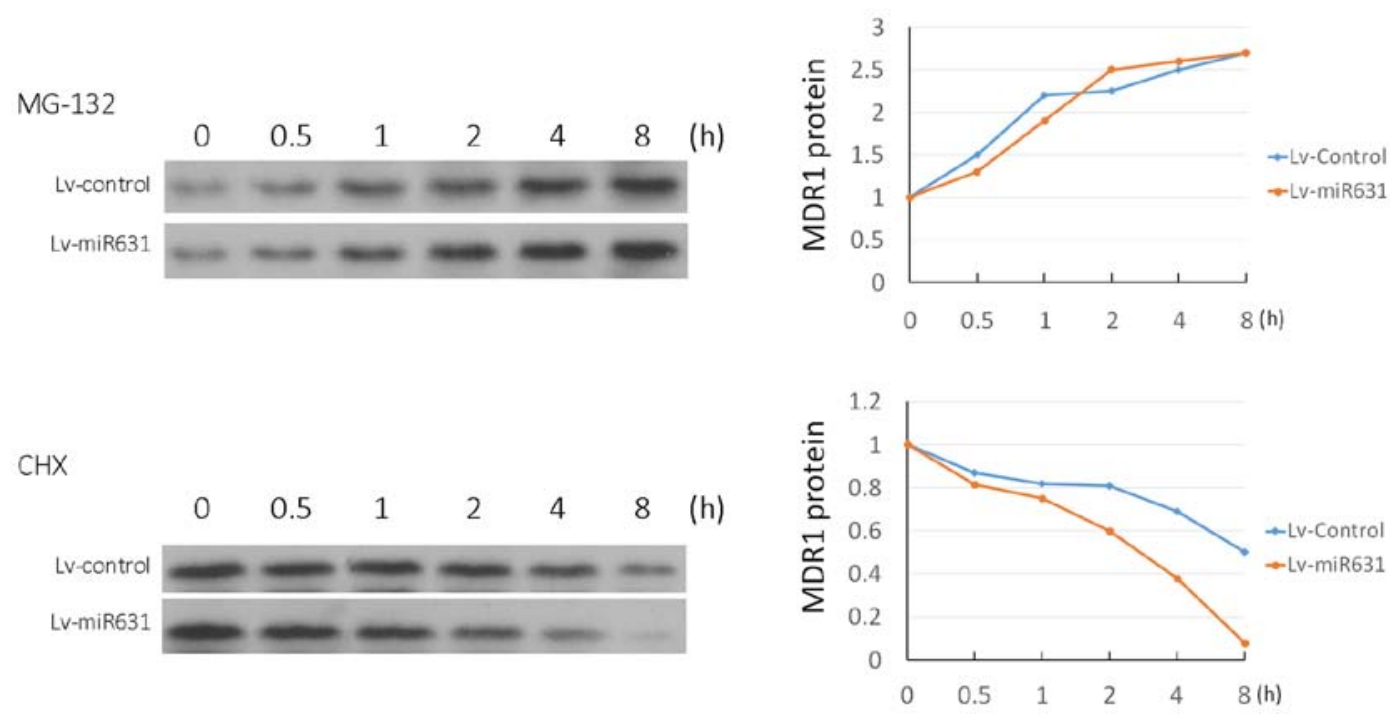

Figure 6. MDR1 protein in U-266/BTZ cells overexpressing miR-631. Seventy-two hours after infection with the indicated lentivirus, U-266/BTZ cells were treated with MG-132 or cycloheximide (CHX) for the indicated length of time and subjected to western blotting for MDR1.

\section{Discussion}

Multiple myeloma (MM) is a common hematologic malignancy, accounting for approximately $10 \%$ of all hematologic malignancies (8). None of the current treatments completely cure MM. Some commonly used treatments for MM are chemotherapy, radiotherapy, hematopoietic stem cell transplantation and novel molecular targeting agents (9). Studies 
have shown that BTZ, a reversible inhibitor of $26 \mathrm{~S}$ proteasome chymotryptic activity, increases drug sensitivity in dexamethasone-, melphalan-, and thalidomide-resistant myeloma cells. This sensitivity increases drug-induced apoptosis in resistant or non-resistant myeloma cells (4). BTZ is the first FDA-approved proteasome inhibitor for clinical treatment of MM, and it has a high specificity and efficiency. With an increase in clinical use of BTZ over time, some patients develop BTZ resistance and experience relapse, ultimately reducing the efficacy of BTZ (10). Therefore, the search for approaches to prevent the development of resistance or eradicate malignant plasma cells has become a hot research topic. To date, there are few studies on BTZ resistance, the mechanism of which remains unknown.

Although chemotherapy is an important cancer treatment, drug resistance creates significant challenges to eliminate cancer cells and cause poor prognosis. Many studies have shown that miRNAs are involved in cancer initiation and development by regulating apoptosis, proliferation, differentiation and metastasis as well as influencing drug resistance via their target genes $(11,12)$. A study of differentially expressed miRNAs and the affiliated regulation of drug resistance-associated genes may contribute to understanding the mechanisms of drug resistance (13). All mutations, misexpression and abnormal processing of miRNAs impair function and can result in abnormal target gene expression. When a target gene is involved in the tumor cell response to drugs, gene chemosensitivity will be changed. Akao et al found that miR-145 and miR-34a improved the sensitivity of DLD-1 cells to 5-Fu (14). A recent study by Bai et al showed that miR-21 is overexpressed in various cancers and is involved in chemoresistance. For example, miR-21 mediates daunorubicin (DNR) resistance in K562 cells, and miR-21 depletion increases the sensitivity of K562/DNR cells to DNR. In addition, miR-21-mediated drug resistance is associated with the PI3k/Akt pathway and the PTEN protein (15). miR-200c regulates the TGF- $\beta$ / ZEB1 pathway via its target ZEB1, resulting in trastuzumab resistance in breast cancer (16). These studies demonstrate that miRNAs are closely related to drug resistance and regulation. Studies indicated that hsa-miR-631, which is located on chromosome 15 , is abnormally expressed in pancreatic cancer and decreases the migration and invasion of pancreatic cancer by inhibiting one of its target genes, ZAP70 (17). However, there are no studies on the association of miR-631 with cancer drug resistance.

Approximately $80-90 \%$ of cell proteins are degraded by the ubiquitin-proteasome pathway (UPP) (18); thus, up to $90 \%$ of cell proteins may be a target for BTZ. The study of abnormal degradation of UPP pathway proteins may facilitate an understanding of drug resistance in MM. UbcH10, also known as UBE2C, is a gene located at 20q13.12 and has a key role in UPP-mediated protein degradation (19). Research has demonstrated that UbcH10 is highly expressed in many types of cancers, including breast cancer, ovarian cancer, thyroid cancer, oesophageal cancer, lymphoma, MM and hepatocellular carcinoma $(20,21)$. In addition, $\mathrm{UbcH} 10$ overexpression is often associated with a high cancer grade, high proliferation and poor tumor prognosis $(22,23)$. There are few studies on the relation between $\mathrm{UbcH} 10$ and chemosensitivity. Zhao et al (24) found that UbcH10 depletion increased the sensitivity of lung cancer cells to 5-Fu. Wang et al (25) found that UbcH10 silencing reversed the cyclophosphamide resistance in breast cancer cell lines. These results suggest that UbcH10 may be a gene therapy target due to an influence on drug resistance. As a key part of UPP-mediated protein degradation, UbcH10 can degrade regulators involved in cell cycle regulation, apoptosis and DNA transcription and repair (26). In the UPP, E1 (ubiquitin activating enzyme) first activates ubiquitin and transfers it to E2 (ubiquitin conjugating enzyme). E2, together with E3 (ubiquitin ligating enzyme), then recognizes the substrate protein. The ubiquitin is then either transferred directly or transferred to E3, where the substrate protein is degraded in the $26 \mathrm{~S}$ proteasome (27). Zhao et al (24) found that drug resistance caused by UbcH10 knockdown may be associated with the MDR1 gene. Further studies on regulatory relationships between UbcH10 and MDR1 have not been conducted. The main objectives of the present study were to determine the association of $\mathrm{UbcH} 10$ and BTZ resistance in myeloma cells and its involvement in MDR1 expression.

We first established BTZ-resistant MM cell lines and found that while UbcH10 protein was higher in the resistant cells than in parental cells, there was no significant difference in UbcH10 mRNA between the groups. This result suggests that the high UbcH10 expression found in resistant cell lines may be due to inactivated post-transcriptional regulation. Therefore, we sought miRNAs that may cause abnormal expression of UbcH10. Using bioinformatic analysis tools, we obtained miRNAs that contain a binding site in the 3'UTR region of the UbcH10 gene and assessed the miRNAs in both resistant cells and their parental cells. The target miRNA hsa-miR-631 was identified by analysis and a correlation of miRNA levels. Luciferase reporter assay verified that hsa-miR-631 may inhibit translation by binding to UbcH10-3'UTR. An increase in UbcH10, stemming from a decrease in miR-631 in BTZ-resistant myeloma cells, may cause BTZ resistance. The overexpression of miR-631 in three BTZ-resistant cell lines reduced BTZ $\mathrm{IC}_{50}$ values. Furthermore, overexpression enabled either resensitization or allowed low BTZ concentrations to induce apoptosis in these cells. We next explored how $\mathrm{UbcH} 10$ expression altered the sensitivity to BTZ. The data showed that UbcH10 was positively related to MDR1 expression, as it inhibits MDR1 ubiquitination. These results collectively suggest the presence of a miR-631/UbcH10/MDR1 pathway during the development of BTZ resistance in MM. In this study, we also verified the specificity of the pathway, as miR-631 overexpression reversed the resistance to BTZ by inhibiting UbcH10 expression while promoting the ubiquitination of MDR1 and reducing MDR1 protein levels. Moreover, overexpression of exogenous UbcH10 under the control of a CMV promoter while free of the miR-631 binding site inhibited the reversion of BTZ resistance through miR-631 overexpression.

Although the present study demonstrated reduced miR-631 in BTZ-resistant MM cell lines for the first time and elucidated that miR-631 regulates MDR1 via UbcH10, there remain two questions to be solved in the development of BTZ resistance in myeloma cells. First, we must ascertain whether BTZ resistance in myeloma cells begins with the low expression of miR-631. Second, studies must be carried out to ascertain how UbcH10 regulates MDR1 expression and whether the regulation is direct or not. Regarding the first question, a possible explanation may be that BTZ induction altered the activity of a certain 
nuclear transcription factor that regulates the transcription of miR-631. Studies have revealed that BTZ resistance is mainly associated with the $\mathrm{NF}-\kappa \mathrm{B}$ pathway, heat-shock proteins or overexpression of BCL family members. We, therefore, plan to study the mechanism upstream of miR-631 by analyzing differentially expressed transcription factors in cells before and after BTZ treatment with a microarray for transcription factors. For the second question, we may come to a deduction from our current knowledge. It is known that $\mathrm{UbcH} 10$ recognizes the substrate and transfers ubiquitin activated by E1 to the target protein, which seems unable to explain the positive correlation we found between UbcH10 and MDR1 in BTZ-resistant cells. In the ubiquitin-proteasome pathway, the target protein may be labelled with K48- or K11-linked polyubiquitin chains. In addition, a previous study showed that polyubiquitin chains comprised of two ubiquitin units result in more effective degradation by proteasome (28). Therefore, we speculate that low expression of UbcH10 (not knockout) tends to produce two-ubiquitin chains, which, compared with fourubiquitin chains, accelerates the degradation of p-glycoprotein. It is only a possible hypothesis. Some E2s play their role in specific cellular process, further study is needed to illustrate all roles of E2s.

In conclusion, we identified that the miR-631/UbcH10/ MDR1 pathway is involved in the development of BTZ resistance in myeloma cells. In addition, in the present study, an increased sensitivity of myeloma cells to BTZ was accomplished by overexpression of miR-631 using genetic engineering. These results may help resolve the mechanisms of BTZ resistance. miR-631 may be used as a genetic marker for the selection of therapy regimes in MM. However, for patients with low miR-631 levels, therapies other than those based on BTZ may be more effective.

\section{Acknowledgements}

This study was supported in part by the National Natural Sciences Fund Project of China (NSFC no. 81470360 , 81372529, 81372543 and 81300391).

\section{References}

1. Barlogie B, Shaughnessy J, Tricot G, Jacobson J, Zangari M, Anaissie E, Walker R and Crowley J: Treatment of multiple myeloma. Blood 103: 20-32, 2004

2. Anderson KC: Therapeutic advances in relapsed or refractory multiple myeloma. J Natl Compr Canc Netw 11 (Suppl 5): 676-679, 2013

3. Accardi F, Toscani D, Bolzoni M, Dalla Palma B, Aversa F and Giuliani N: Mechanism of action of bortezomib and the new proteasome inhibitors on myeloma cells and the bone microenvironment: impact on myeloma-induced alterations of bone remodeling. BioMed Res Int 2015: 172458, 2015.

4. Adam Z, Sčudla V, Krejčí M, Cermáková Z, Pour L and Král Z: Treatment of AL amyloidosis in 2012; the benefit of new drugs (bortezomib, thalidomide, and lenalidomide). Summary of published clinical trials. Vnitr Lek 59: 37-58, 2013 (In Czech).

5. Adachi Y, Yoshio-Hoshino N and Nishimoto N: Gene therapy for multiple myeloma. Curr Gene Ther 8: 247-255, 2008.

6. Tétreault N and De Guire V: miRNAs: Their discovery, biogenesis and mechanism of action. Clin Biochem 46: 842-845, 2013.

7. Parker JS, Roe SM and Barford D: Molecular mechanism of target RNA transcript recognition by Argonaute-guide complexes Cold Spring Harb Symp Quant Biol 71: 45-50, 2006.
8. Abe M: Multiple myeloma. Nihon Rinsho 67: 991-995, 2009 (In Japanese).

9. Abdi J, Chen G and Chang H: Drug resistance in multiple myeloma: Latest findings and new concepts on molecular mechanisms. Oncotarget 4: 2186-2207, 2013.

10. Abidi MH, Gul Z, Abrams J, Ayash L, Deol A, Ventimiglia M, Lum L, Mellon-Reppen S, Al-Kadhimi Z, Ratanatharathorn V, et al: Phase I trial of bortezomib during maintenance phase after high dose melphalan and autologous stem cell transplantation in patients with multiple myeloma. J Chemother 24: 167-172, 2012.

11. Abba M, Mudduluru G and Allgayer H: MicroRNAs in cancer: Small molecules, big chances. Anticancer Agents Med Chem 12: 733-743, 2012.

12. Azmi AS, Bao B and Sarkar FH: Exosomes in cancer development, metastasis, and drug resistance: A comprehensive review. Cancer Metastasis Rev 32: 623-642, 2013.

13. Allen KE and Weiss GJ: Resistance may not be futile: microRNA biomarkers for chemoresistance and potential therapeutics. Mol Cancer Ther 9: 3126-3136, 2010.

14. Akao Y, Khoo F, Kumazaki M, Shinohara H, Miki K and Yamada N: Extracellular disposal of tumor-suppressor miRs-145 and -34a via microvesicles and 5-FU resistance of human colon cancer cells. Int J Mol Sci 15: 1392-1401, 2014.

15. Bai H, Xu R, Cao Z, Wei D and Wang C: Involvement of miR-21 in resistance to daunorubicin by regulating PTEN expression in the leukaemia K562 cell line. FEBS Lett 585: 402-408, 2011.

16. Bai WD, Ye XM, Zhang MY, Zhu HY, Xi WJ, Huang X, Zhao J, Gu B, Zheng GX, Yang AG, et al: MiR-200c suppresses TGF- $\beta$ signaling and counteracts trastuzumab resistance and metastasis by targeting ZNF217 and ZEB1 in breast cancer. Int J Cancer 135: 1356-1368, 2014.

17. Fu D, Liu B, Zang LE and Jiang H: MiR-631/ZAP70: A novel axis in the migration and invasion of prostate cancer cells. Biochem Biophys Res Commun 469: 345-351, 2016.

18. Eldridge $A G$ and $\mathrm{O}^{\prime} B r i e n ~ T:$ Therapeutic strategies within the ubiquitin proteasome system. Cell Death Differ 17: 4-13, 2010.

19. Okamoto Y, Ozaki T, Miyazaki K, Aoyama M, Miyazaki M and Nakagawara A: UbcH10 is the cancer-related E2 ubiquitin-conjugating enzyme. Cancer Res 63: 4167-4173, 2003.

20. Lin J, Raoof DA, Wang Z, Lin MY, Thomas DG, Greenson JK, Giordano TJ, Orringer MB, Chang AC, Beer DG, et al: Expression and effect of inhibition of the ubiquitin-conjugating enzyme E2C on esophageal adenocarcinoma. Neoplasia 8: 1062-1071, 2006.

21. Troncone G, Guerriero E, Pallante P, Berlingieri MT, Ferraro A, Del Vecchio L, Gorrese M, Mariotti E, Iaccarino A, Palmieri EA, et al: UbcH10 expression in human lymphomas. Histopathology 54: 731-740, 2009.

22. Berlingieri MT, Pallante P, Sboner A, Barbareschi M, Bianco M, Ferraro A, Mansueto G, Borbone E, Guerriero E, Troncone G, et al: UbcH10 is overexpressed in malignant breast carcinomas. Eur J Cancer 43: 2729-2735, 2007.

23. Berlingieri MT, Pallante P, Guida M, Nappi C, Masciullo V, Scambia G, Ferraro A, Leone V, Sboner A, Barbareschi M, et al: $\mathrm{UbcH} 10$ expression may be a useful tool in the prognosis of ovarian carcinomas. Oncogene 26: 2136-2140, 2007.

24. Zhao L, Jiang L, Wang L, He J, Yu H, Sun G, Chen J, Xiu Q and $\mathrm{Li}$ B: UbcH10 expression provides a useful tool for the prognosis and treatment of non-small cell lung cancer. J Cancer Res Clin Oncol 138: 1951-1961, 2012.

25. Wang C, Pan YH, Shan M, Xu M, Bao JL and Zhao LM: Knockdown of UbcH10 enhances the chemosensitivity of dual drug resistant breast cancer cells to epirubicin and docetaxel. Int J Mol Sci 16: 4698-4712, 2015.

26. Doherty FJ, Dawson S and Mayer RJ: The ubiquitin-proteasome pathway of intracellular proteolysis. Essays Biochem 38: 51-63, 2002.

27. Summers MK, Pan B, Mukhyala K and Jackson PK: The unique $\mathrm{N}$ terminus of the UbcH10 E2 enzyme controls the threshold for APC activation and enhances checkpoint regulation of the APC. Mol Cell 31: 544-556, 2008.

28. Stieglitz B, Rana RR, Koliopoulos MG, Morris-Davies AC, Schaeffer V, Christodoulou E, Howell S, Brown NR, Dikic I and Rittinger K: Structural basis for ligase-specific conjugation of linear ubiquitin chains by HOIP. Nature 503: 422-426, 2013. 